\title{
THE HYALINE MEMBRANE SYNDROME IN PREMATURE INFANTS
}

\author{
BY \\ WILLIAM BLYSTAD \\ From the Children's Department, Rikshospitalet, Oslo
}

(RECEIVED FOR PUBLICATION AUGUST 3, 1955)

In recent years attention has been drawn to the frequent occurrence in premature infants of a characteristic clinical picture of dyspnoea setting in a few hours after birth. This condition, which is also observed in full-term infants, will be called here 'early neonatal dyspnoea'. Investigations in full-term infants presenting this clinical picture have produced evidence of an increased minute volume and considerably increased respiratory effort (Karlberg, 1954). Blood gas analyses carried out on premature infants presenting the same picture have shown, notwithstanding increased respiratory effort, marked respiratory failure, which must be of pulmonary origin and causes a pronounced acidaemia, and seems to be the principal cause of death in the fatal cases (Blystad, 1955). It has been held that this clinical picture bears a relationship to the development of hyaline membrane and atelectases in the lungs, 'the hyaline membrane syndrome' (Parmelee, 1952; Miller and Behrle, 1953; Clifford, 1953; Arey and Dent, 1953; Potter, 1952, 1953; M. \& R. Dietetic Laboratories, 1953).

The object of the present research was to test this hypothetical aetiological relationship.

\section{Materials}

The study is based on a clinical and pathological study of 47 premature infants who died in the course of the early neonatal period. Post-mortem examinations were carried out in the pathological laboratory of the hospital.

\section{Results}

According to the clinical picture the material has been classified into two groups. The first of these groups includes 25 premature infants presenting the typical clinical picture of early neonatal dyspnoea; 11 of these patients also had one or more attacks of apnoea, and in four the dyspnoea gradually changed into a protracted stage of respiratory depression, lasting several hours, before death.
All the patients in this group died within the first four days of life.

The second group comprises 22 patients who showed no sign of early neonatal dyspnoea. Instead, respiration, which was slow and gasping, was depressed, and in 18 there were protracted attacks of apnoea. Death occurred in the course of the first five days of life.

Convulsions or a bulging fontanelle, indicating a severe intracranial haemorrhage, were observed in none of the 47 cases.

Tables 1 and 2 show the pulmonary and intra-

TABLE 1

PULMONARY NECROPSY FINDINGS IN 47 PREMATURE INFANTS DYING IN THE EARLY NEONATAL PERIOD

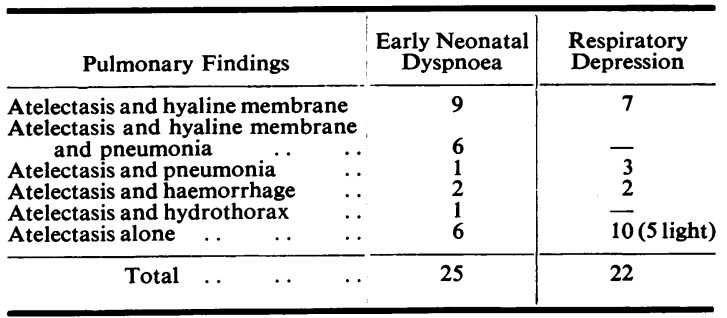

TABLE 2

INTRACRANIAL PATHOLOGICAL FINDINGS IN 47 PREMATURE INFANTS DYING IN EARLY NEONATAL PERIOD AFTER MARKED RESPIRATORY COMPLICATIONS

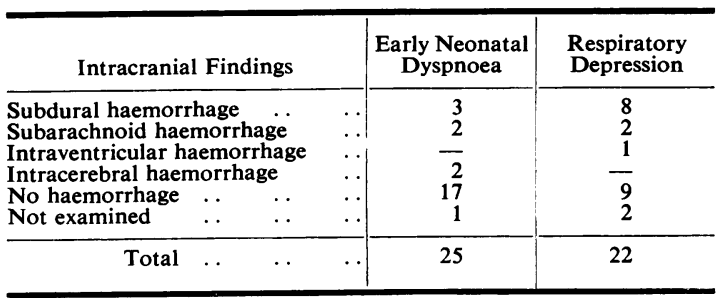

cranial findings revealed by the necropsy. The patients in the first group showed marked pulmonary changes, and extensive atelectases were seen in all the cases. Hyaline membrane was found 
in 15 of the 25 infants. In four cases there was a relatively severe pneumonia, and in the three others the pneumonic foci were few and scattered. In this group the intracranial haemorrhage in four of seven cases seemed to have been moderate.

In the second group obvious abnormal pulmonary findings were also noted in most cases. In five cases, however, the only finding was a fairly moderate atelectasis. Seven of the 22 patients had hyaline membrane in the lungs. Intracranial haemorrhage was somewhat more frequent in this group, which in several cases was more extensive than in the first group.

\section{Discussion}

In 15 of the first group of 25 infants presenting with early neonatal dyspnoea necropsy revealed hyaline membrane and pulmonary atelectasis. In the remaining 10 cases presenting with the same clinical picture, hyaline membrane was absent but there were significant pulmonary changes in the form of marked atelectasis, pneumonia or haemorrhage. These findings suggest that though the clinical picture of early neonatal dyspnoea indicates the presence of marked pulmonary changes it does not give certain information as to their nature. Most frequently, however, massive atelectasis will dominate the picture. The cause of the atelectasis and the significance of the hyaline membrane are not yet clear.

In the second group of 22 premature infants the clinical picture was characterized by a depressed respiration which might seem to indicate a damaged central nervous system. Nevertheless, in seven of these patients the necropsy revealed hyaline membrane and atelectasis. Thus, these pathological changes are often present also in cases where no dyspnoea has been observed. Significant pulmonary findings in the form of atelectasis, pneumonia or haemorrhage were also seen in many of the other patients of the second group. An evaluation of the significance of the pulmonary findings will be difficult. It is possible that they are mainly secondary to a central injury with depression of the respiration and asphyxia. Intracranial haemorrhage was relatively frequent in this group. However, in nine cases there was no haemorrhage, and the origin of the respiratory depression will have to be sought elsewhere.
The absence of dyspnoea does not, of course, exclude the possibility that the pulmonary changes were the primary cause of the asphyxia in some of the infants of the second group also. The poorly functioning lungs could have led to anoxic depression of the respiratory centre, which could then no longer respond adequately to the respiratory situation. Such a development was easily discernible in several of the first group of infants with early neonatal dyspnoea, in whom, after an initial stage of increased respiratory effort, the picture changed into a relatively protracted phase characterized by markedly depressed respiration. I believe that in many premature infants pulmonary pathological changes constitute the main cause of asphyxia even where no early neonatal dyspnoea has been observed.

\section{Summary}

The clinical data and the necropsy findings of 47 premature infants who died in the course of the early neonatal period were examined. Early neonatal dyspnoea was observed in 25 patients. In all cases there was extensive atelectasis. In 15 of these the necropsy revealed hyaline membrane in the lungs. The other patients with this clinical picture also showed marked pulmonary changes. The clinical picture reflected a pulmonary pathology of varying character, in which marked atelectasis of unknown origin was a constant finding.

In 22 patients respiration was depressed centrally. In these intracranial haemorrhage was found somewhat oftener but was not a precondition to the establishment of this clinical picture. In this group considerable pathological changes were also frequently found in the lungs. In seven cases the necropsy revealed hyaline membrane. It is probable, in spite of the lacking dyspnoea, that pulmonary changes were the main cause of the asphyxia in some of the patients in the second group.

\section{REFERENCES}

Arey, J. B. and Dent, J. (1953). J. Pediat., 42, 205

Blystad, W. (1955). Acta paediat. Uppsala. To be published.

Clifford, S. H. (1953). J. Pediat., 43, 237.

Karlberg, P. (1954). The Proceedings of the Eleventh Northern Paediatric Congress. Oslo, 1954. To be published.

Miller, H. C. and Behrle, F. C. (1953). Pediatrics, 12, 141.

M. \& R. Dietetic Laboratories (1953). Pulmonary Hyaline Membranes: Report of the Fifth M. \& R. Pediatric Research Conference, 1952. Columbus, Ohio.

Parmelee, A. H. (1952). J. Pediat., 41, 591.

Potter, E. (1952). Pathology of the Fetus and the Newborn. Chicago. - (1953). Advanc. Pediat., 6, 157. 